\title{
Electrochemical nanobiosensors and protein detection
}

Martina Zamuner ${ }^{1}$, Stefano Pozzi Mucelli², Massimo Tormen33, Giorgio Stanta²*, Paolo Ugo ${ }^{1 *}$ (DOI 10.3884/0001.8)

\begin{abstract}
Nanostructured electrochemical biosensors are prepared by immobilizing the biorecognition elements on the polycarbonate (PC) surrounding gold nanodisks (approximately, $30 \mathrm{~nm}$ in diameter) in nanoelectrode ensembles (NEEs) made in track-etched commercial membranes. A suitable redox mediator is added to the sample solution to shuttle electrons from the nanoelectrodes to the biorecognition layer, both elements being in strict spatial proximity. In this way one can exploit the highly improved signal-to-background current ratio which is peculiar of NEEs with respect to other electrochemical transducers. Two detection schemes were tested: one based on the direct immobilization of the target protein on the PC of the NEE (approach A) and the other based on the immobilisation on PC of an antibody to capture the target protein (approach B). In both cases, the biorecognition process was completed by adding a primary antibody and a secondary antibody with horse radish peroxidase (HRP) as enzyme label; methylene blue was the redox mediator added to the electrolyte solution. Typical target analytes were single chain fragment variable proteins, for approach A, and trastuzumab (also known as Herceptin ${ }^{\circledR}$ ), for approach B. NEE-based capture sensors were tested successfully to detect small amounts of the receptor protein HER2 in biological samples.

Stanta G. Eur J Nanomed 2008; 1:33-36
\end{abstract}

Keywords: Nanoelectrode, electrochemical biosensor, proteomics, trastuzumab, voltammetry.

\section{Introduction}

Biosensors are self-contained devices able to provide chemical or biological information as a signal (typically, optical or electrical) generated by a transducer in intimate contact with a biorecognition layer. (1) In electrochemical sensors, a change in the redox state of the biorecognition/analyte system related to the biorecognition event, produces a change in an electrochemical quantity which can be monitored by classical electroanalytical techniques. Electrochemical systems can be miniaturized below the micrometer scale, using individual nanoelectrodes or ordered arrays or random ensembles of nanoelectrodes (NEA and NEE, respectively). (2) In particular, the use of NEAs and NEEs allows to overcome problems related to the requirement for high signal amplification and careful shielding of electrical noise which is required for measurements with individual nanoelectrodes. Signals given by NEAs and NEEs are indeed the summation (weighed by diffusion effects) of the signals generated at each single nanoelectrode element; (3) a drawback is that NEAs and NEEs do not allow the extreme "nano-turization" degree achievable with individual nanoelectrodes, however they can be brought down to arrays of overall microsize, but made of nanometer sized single components (4).

Among the others, NEEs prepared by controlled deposition of metal nanoelements using polycarbonate ultrafiltration membranes as template, are attractive and increasingly used, since their fabrication procedure is easy and accessible. (5) Electroless deposition of gold in polycarbonate templates for producing NEEs was introduced some years ago by Menon and Martin (3) and refined more recently in our laboratories. (6) Membranes with pore diameter from $10 \mathrm{~nm}$ to $10 \mu \mathrm{m}$ are commercially available. The diameter of the pores in the template determines the diameter of the Au-nanodisk elements that make up the NEE while the density of the pores in the membrane determines the number of Au- disk nanoelectrodes per $\mathrm{cm}^{2}$ and their average distance. A NEE is an assembly of a very large number of very small ultramicroelectrodes confined in a rather small space (108 electrodes $/ \mathrm{cm}^{2}$ ). NEEs can exhibit distinct voltammetric response regimes depending on the scan rate or distance between the nanoelectrode elements. (3) The total overlap (TO) regime is operative when radial diffusion boundary layers at each nanoelectrode overlap (slow scan rates and/or small distance between nanoelectrodes); this is the diffusion regime commonly observed for NEEs fabricated from commercial track-etched membranes. Under these conditions, the signal obtained at a NEE (namely, the faradaic peak current) is proportional to the geometric area (Ageom; that is area of the nanodisc electrodes plus insulating membrane between them), while the background current (i.e. double layer charging current) depends on the active area (Aact; area of the metal nanoelectrode elements alone) (4). As a consequence of the high Ageom/ Aact ratio, NEEs are characterized by detection limits 2-3 orders of magnitude lower than those achievable with regular electrodes of the same geometric area. $(7,8)$ Some bioanalytical applications of arrays and ensembles of nanoelectrodes have been very recently reviewed. (2)

In typical schemes used to obtain electro-chemical biosensors, a biorecognition layer is directly immobilized on
1 Department of Physical Chemistry, University of Venice, Santa Marta 2137, 30123 Venice, Italy

2 Department of Clinical, Morphological and Technological Sciences, University of Trieste, Strada di Fiume 447, 34149 Trieste, Italy and Molecular Histopathology Laboratory, International Centre for Genetic Engineering and Biotechnology, Trieste, Italy

3 Laboratorio Nazionale TASC-INFM-CNR, Area Science Park, S.S. 14, Km. 163.5, I-34012 Trieste, Italy

Corresponding authors: stanta@icgeb.org (G.Stanta); ugo@unive.it (P.Ugo) 


\section{Clinical trials}

the electrode surface and the signal is produced by exchange of electrons between this layer and the underlying electrode. However, in the case of miniaturized electrodes, such as in the case of nanoelectrodes, the amount of immobilized biomolecules can be too small to give signals exploitable for analytical purposes. In order to increase the electrode area available for the immobilization, the template membrane can be etched. (9-11) In this way, 2D ensembles of metal nanodisks turn into a $3 \mathrm{D}$ ensembles of nanowires. However, this causes the loss of the main analytical advantage of NEEs, that is improved signal-to-background current ratios. (11) In a recent paper (12) we demonstrated the feasibility and the advantages of a different approach, that is the immobilization of the biorecognition elementonthepolycarbonatesurrounding the nanodisk electrodes and not on the nanoelectrodes themselves. In such a design, transducer and biorecogniton layer are not overlapped, but integrated in strict proximity; since no increase in the Aact is required, voltammetric signals are produced at the highest signal/ background current ratio.

In the present study we report on the use of these nanobiosensors to determine two target proteins. One is the single chain fragment variable (ScFV) protein chosen as a general model protein, the other is the HER2 receptor which represents a quite important target protein connected to the development of the so-called personalized therapies. The possibility to detect HER 2 is indeed extremely important for the identification of cancer that can be treated successfully with Herceptin (Trastuzumab), a recombinant DNA-derived humanized murine monoclonal antibody directed against the extracellular domain of the $\mathrm{HER} 2$ receptor.

\section{Experimental section \\ Apparatus and materials}

All electroanalytical measurements were carried out at room temperature $\left(22 \pm 1^{\circ} \mathrm{C}\right)$ under a nitrogen atmosphere, using a three-electrode single-compartment cell equipped with a platinum coil counter electrode and an $\mathrm{Ag} / \mathrm{AgCl}(\mathrm{KCl}$ saturated) reference electrode. All potential values are referred to this reference electrode. A $\mathrm{CH} 660 \mathrm{~A}$ potentiostat controlled via PC by its own software was used for voltammetric measurements. All the used chemicals were reagent grade and utilized without further purification. Horseradish peroxidase, type VI, 298 Purpurogallin units/mg solid, was purchased from Sigma. Purified water was obtained using a Milli-Ro plus Milli-O (Millipore) water purification system.

NEEs were prepared by template gold electroless deposition (13), following recently described updates in the procedure. $(6,13)$ The templating membranes were polycarbonate tracketched filter membranes (SPI-pore, 47 $\mathrm{mm}$ filter diameter, $6 \mu \mathrm{m}$ thickness) with a nominal pore diameter of $30 \mathrm{~nm}$, average pore density $6 \times 108$ pore $/ \mathrm{cm}^{2}$ and coated by the producer with the wetting agent polyvinylpyrrolidone.

A hole punched in insulating layer of plastics (Monokote film by Topflite, that is polyethylene terephtalate film with a polyethylene layer which acts as heat activated adhesive) determined the Ageom value, typically $0.07 \mathrm{~cm}^{2}$. The value of Aact, estimated by SEM analysis, was $0.001 \mathrm{~cm}^{2}$.

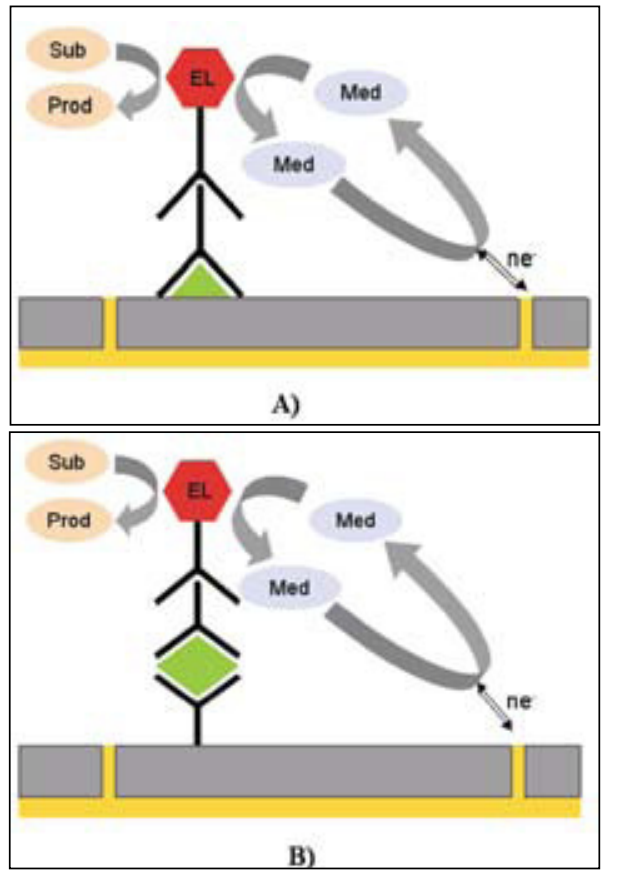

Figure 1. Schematic illustration of the two detection approaches used with NEEs: (A) the target protein is immobilized on the polycarbonate membrane, then it binds a primary antibody and secondary antibody with the enzyme label (EL); a soluble mediator (Med) shuttle electrons from the nanoelectrode to EL. (B) A primary antibody, specific for the target protein, is first immobilized on the membrane, in order to capture the target protein. The other components follow as in scheme A.
Detection schemes and immobilization procedures

The two detection strategies summarized in Fig. 1 were used. In approach A, shown in Fig. $1 A$, the target protein is directly immobilized on the polycarbonate and recognized and detected by a suitable primary antibody and then by a secondary antibody labelled with HRP.

According to approach $B$, sketched in Fig. 1 B, A specific antibody is immobilized on the polycarbonate, thus capturing the protein of interest, which is finally detected via primary plus secondary labelled antibody.

Single chain fragment variable (ScFv) antibodies were cloned in the vector pDAN 5 , purified from $\mathrm{DH}_{5} \alpha \mathrm{E}$. coli cells using a nickel affinity resin and diluted in bicarbonate buffer (14). A microvolume of $0.5 \mu \mathrm{l}$ of a $4 \mu \mathrm{M}$ ScFv solution was spotted onto the NEE, then incubated for 4 hours at $4^{\circ} \mathrm{C}$. Electrodes were then washed with PBST (Phosphate Buffer Saline, added with $0,2 \% \mathrm{v} / \mathrm{VT}$ Tween 20), and incubated for 10 minutes in $\mathrm{NaBH}_{4}$ to block remaining carboxylic groups. (15)

Then the NEE was incubated with an anti-SV 5 antibody (in house purified, diluted 1:3000 in Phosphate Buffer Saline added with milk powder and Tweenzo (PBST-milk), kindly provided by Daniele Sblattero), and finally with an antimouse-HRP secondary antibody (Dako Cytomation, diluted 1:10000 in PBSTmilk). Trastuzumab functionalised NEEs (T-NEE) were prepared by incubating on the NEE $2 \mu \mathrm{l}$ of $0.5 \mu \mathrm{g} / \mu \mathrm{l}$ trastuzumab (Hercerptin ${ }^{\circledR}$, Genentech, South San Francisco, $(A)$ in carbonate buffer, for 2 hours at $4^{\circ} \mathrm{C}$. After washing with PBST, 10 $\mu \mathrm{l}$ of four dilutions $(1: 1,1: 5,1: 10,1: 50)$ of a $10 \mu \mathrm{g} / \mu \mathrm{l} \mathrm{SKBR}_{3}$ lysate were incubated with the T-NEE for 4 hours at $4^{\circ} \mathrm{C}$; the monoclonal antibody CB-11, diluted 1:5000 in PBST-milk, was used to detect bound Her2, together with an anti-mouse HRP-conjugated antibody.

NEEs used as negative control were not incubated with the cell lysate, but, right after the immobilization of trastuzumab they were incubated with the $C B-11$ antibody and the secondary antibody.

\section{Results and discussion}

Preliminary tests performed in homogeneous solutions (mediator, enzyme and substrate all dissolved in solution), showed that the best mediator suitable to shuttle electron from the Aunanoelectrodes of a NEE to HRP was 
methylene blue (MB) which is reduced reversibly at the NEE according to the following reaction:

$$
12 \mathrm{MB}+2 \mathrm{e}+\mathrm{H}^{+} \longrightarrow \mathrm{LB}
$$

where LB is the leuco (reduced) form of MB. In the presence of HRP and its substrate $\mathrm{H}_{2} \mathrm{O}_{2}, \mathrm{MB}$ shuttles electrons from the nanoelectrode to the enzyme label being involved in $\mathrm{f}$ the following electrocatalytic cycle:

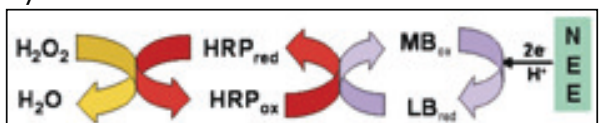

In order to test the efficiency of the detection scheme shown in Fig. 1a, further tests were performed by immobilizing ScFv on the NEE surface. The protein was then bound to anti-SV 5 antibody plus anti-mouse-HRP secondary antibody. The tests were performed by adding increasing concentration of $\mathrm{H}_{2} \mathrm{O}_{2}$, thus allowing one to determine also the best operative range for the concentration of the enzyme substrate. As shown in Fig. 2 , even at relatively low concentration of $\mathrm{H}_{2} \mathrm{O}_{2}$ (namely, $1.5 \mathrm{mM}$ ) the voltammetric patterns assumed the typical sigmoidal electrocatalytic shape (full line).

These results confirmed the feasibility at NEE of the detection scheme $1 \mathrm{~A}$, at least when using protein samples (like the ScFv sample tested here) very rich in analyte. Methylene blue signals (see broken line in Fig. 2) at the NEE functionalised with ScFvanti-SV5-anti-mouse-HRP (see broken line in Fig. 3) did not show significant differences with the signal recorded at unmodified NEEs, so indicating no inhibition by the immobilized proteins on the charge transfer at the surface of the gold nanoelectrodes. The above evidences support the feasibility at NEEs of the detection scheme $1 \mathrm{a}$, at least when high concentration of the target protein are present in the sample, as in the ScFv case.

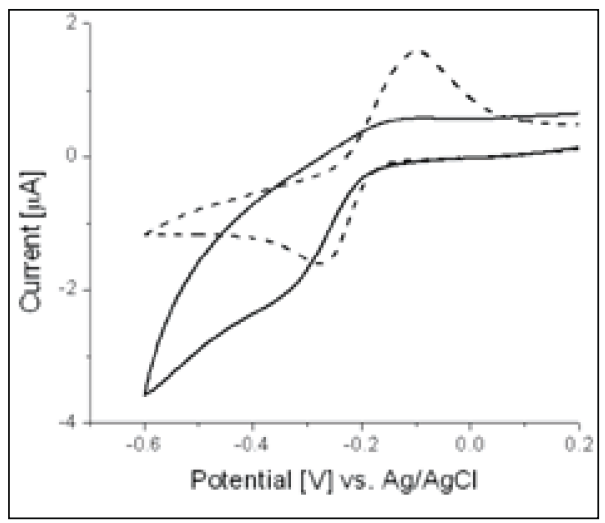

However, to the goal of analysing with NEEs samples containing small concentration of the target protein, the development of capture arrays based on the scheme in Fig.1b appeared more suitable.

To this aim, we tested as capture agent trastuzumab, which is a monoclonal humanized antibody used in the adjuvant therapy of breast cancer. (16) At first, preliminary immunoprecipitation assays were performed. As negative control, we immunoprecipitated our sample using an anti-PCNA (Proliferating cell nuclear antigen, Santa Cruz Biotechnology, CA) antibody, while as positive control, $70 \mu \mathrm{g}$ of whole $\mathrm{SKBR}_{3}$ (17) lysate were loaded onto the gel. Western blot analysis performed after the immunoprecipitation, indicated the bands, at $185 \mathrm{kDa}$ and $95 \mathrm{kDa}$, both due to the Her2 protein, were clearly visible, so confirming (18) the efficiency of trastuzumab as capture agent in vitro. This prompted us to undergo the study of trastuzumab functionalised NEEs. The tests were performed with NEEs functionalized with Trastuzumab (see experimental section). The mediator, namely $0.1 \mathrm{mM} \mathrm{MB}$, and the substrate (1.5 $\mathrm{mM} \mathrm{H}_{2} \mathrm{O}_{2}$ ) were added to the PBS electrolyte. The cyclic voltammetric patterns for the mediator MB at a NEE after the immobilization of trastuzumab, recorded in the absence and in the presence of cell lysate, but always without HER2, overlap each other. This confirms that no change in the voltammetric behaviour of MB at the NEE is caused by functionalization with trastuzumab. Moreover, no aspecific adsorption of the labeled antibody occurs if the target protein (HER2) is not present in the sample.

On the other hand, a significant increment in the current intensity $(\mu \mathrm{l})$ was observed after incubation in the cell lysates, containing the target protein HER2, followed by incubation with

Fig. 2. Cyclic voltammograms recorded at a NEE functionalised with the target protein ScFv, the primary anti-SV 5 antibody and the anti-mouse-HRP secondary antibody; in solution containing $0.1 \mathrm{mM}$ methylene blue before (dash line) and after adding 1.5 $\mathrm{mM} \mathrm{H}_{2} \mathrm{O}_{2}$ (full line). Scan rate: 50 $\mathrm{mV} / \mathrm{s}$, supporting electrolyte $10 \mathrm{mM}$ phosphate buffer, $\mathrm{pH} 7$. the primary antibody and secondary antibody with the HRP label. Some tests were performed by changing the HER2 concentration in the sample by proper dilution of a cell lysate $(2 \mathrm{ng} / \mu$ l before dilution) standardized by Western blot analysis, with a HER 2 content of $1 \mu \mathrm{g} /$ $\mu \mathrm{l}$, before dilution. The content of total proteins in the same undiluted sample was $10 \mu \mathrm{g} / \mu \mathrm{l}$. The immobilization of the all four-proteins chain (specific antibody, target protein, primary antibody and secondary labeled antibody) hinders slightly, but do not block the electron transfer from the Au-nanoelectrodes to the mediator. This suggests that the protein chain is immobilized in very close proximity to the gold nanodisks, but not directly on them. The voltammogram recorded in the presence of $1.5 \mathrm{mM} \mathrm{H}_{2} \mathrm{O}_{2}$ shows a typical electrocatalytic character, with electrocatalytic $\mu \mathrm{l}$ values which increase with the HER 2 concentration as shown in Fig. 3. The concentration of $\mathrm{H}_{2} \mathrm{O}_{2}$ here used corresponds to the value which gives the highest electrocatlytic increase in peak currents, without causing an undesired increase in background current, as observed by us for instance when 3 or 5 $\mathrm{mM} \mathrm{H}_{2} \mathrm{O}_{2}$ was added to the sample.

Note that the sensitivity of classical immunochemical assays, such as Western blotting, is not high enough to detect the HER2 analyte at the dilution levels of our 1:10 or 1:50 samples, while good electrocatalytic signals were detected with the NEE in these diluted samples.

Preliminary tests on lysates of cancer cells overexpressing $\mathrm{HER}_{2}$ were successful and encouraging for the application of the NEE based biosensor to analyses of clinical samples. (12)

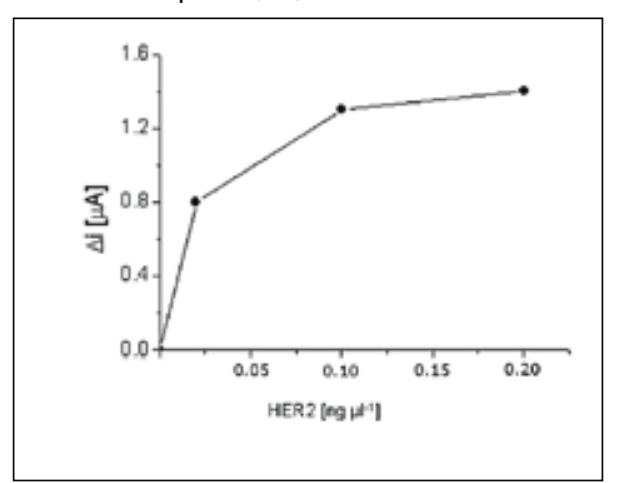

Figure 3. Dependence of electrocatalytic current increase $(\Delta \mathrm{l})$ on HER 2 concentration at a NEE functionalized with trastuzumab. Cell lysates con-taining the target protein were diluted 1:10 (full line) and 1:50 (dashed line). 


\section{Clinical trials}

\section{Conclusions}

The results presented here confirm the potentiality of electrochemical nanobiosensors based on ensembles of nanoelectrodes for the sensitive determination of target proteins, since with NEEs it was possible to detect target proteins in rather diluted samples (at least down to $20 \mathrm{pg} / \mu \mathrm{l}$ ), where traditional, immunochemicalmethod, suchasWestern blotting, failed for lack of sensitivity. Further studies aimed to validate the analytical performances of the sensor in terms of detection limit, dynamic range, accuracy and precision, are in progress. In combination with the possibility to prepare singly addressable groups of NEEs, (19) the present approach can open new prospects towards the development of miniaturized arrays of nanolectrodes, for multi-protein determinations.

\section{Acknowledgements}

Financial support by MUR (Rome), PRIN 2006, is gratefully acknowledged.

\section{References}

1 Cunningham A.J., Introduction to Bioanalytical Sensors, J. Wiley \& Sons, New York, 1998.

2 Shi H.B., Yeh J.I., Nanomedicine , 2007, 2, 587-598

3 Menon, V.P.; Martin, C.R. Anal. Chem. 1995, 67, 1920-1928.

4 Moretto L.M., Pepe N., Ugo P , Talanta 2004, 62, 1055-1060

5 Ugo, P.; Pepe, N.; Moretto, L.M.; Battagliarin, M. J. Electroanal. Chem. 2003, 560, 51.

6 Pereira, F.C.; Moretto, L.M.;M. De Leo, M.; Boldrin Zanoni, M.V.; Ugo, P. Anal. Chim. Acta 2006, 575, 16-24.

7 Ugo, P.; Moretto, L.M.; Bellomi, S.; Menon, V.P.; Martin, C.R. Anal. Chem. 1996, 68, 4160-4165.

8 Ugo, P.; Moretto, L.M.; Vezzà, F. ChemPhysChem 2002, 3, 917-925. $9 \mathrm{Yu}, \mathrm{S}$.; Li, N.; Wharton, J.; Martin, C.R. Nano Lett. 2003, 3, 815-818.10 De Leo, M. ; Kuhn, A. ; Ugo, P. Electroanalysis 2007, 19, 227-236.11 Lapierre-Devlin, M.A.; Asher, C.L., Taft, B.J., Gasparac, R., Roberts, M.A., Kelley, S.O. Nano Lett. 2005, 5, 1051-1055.12 S. Pozzi Macelli, M. Zamuner, M. Tormen, G.Stanta, P.Ugo, Biosens. Bioelectron., 2008, in press, doi: 10.1016/j.bios.2008.02.2713 Ugo, P.; Moretto, L. M. In Handbook of Electrochemistry; Zoski, C. G., Ed.; Elsevier: Amsterdam, 2007; Chapter 16, Section 16.2, pp. 678-709.14 Sblattero,
D.; Bradbury, A.; Nat. Biotechnol. 2000, 18, 75-8015 Afanassiev, V.; Hanemann V.; Wolfl, S.; Nucleic Acids Res. 2000, 15, e6616 Simonds, H.M,; Miles, D. Expert Opin Biol Ther. 2007, 7, 487-9117 Pasleau, F.; Grooteclaes, M,; Gol-Winkler, R.

Oncogene 1993, 8, 849-5418 Molina, M.A.; Codony-Servat J.; Albanell, J.; Rojo, F.; Arribas, J.; Baselga, J. Cancer Res. 2001, 61, 4744-9. 19 Zoski, C. G.; Yang, N.; He, P.; Berdondini, L.; Koudelka-Hep, M. Anal. Chem . 2007, 79, 1474-1484. 